IJMMS 2003:1, 15-26

PII. S016117120311037X

http://ijmms.hindawi.com

(c) Hindawi Publishing Corp.

\title{
LOCAL STABILITY OF THE ADDITIVE FUNCTIONAL EQUATION AND ITS APPLICATIONS
}

\author{
SOON-MO JUNG and BYUNGBAE KIM
}

Received 5 October 2001

\begin{abstract}
The main purpose of this paper is to prove the Hyers-Ulam stability of the additive functional equation for a large class of unbounded domains. Furthermore, by using the theorem, we prove the stability of Jensen's functional equation for a large class of restricted domains.
\end{abstract}

2000 Mathematics Subject Classification: 39B82, 39B62.

1. Introduction. The starting point of studying the stability of functional equations seems to be the famous talk of Ulam [14] in 1940, in which he discussed a number of important unsolved problems. Among those was the question concerning the stability of group homomorphisms: let $G_{1}$ be a group and let $G_{2}$ be a metric group with a metric $d(\cdot, \cdot)$. Given $\varepsilon>0$, does there exist a $\delta>0$ such that if a mapping $h: G_{1} \rightarrow G_{2}$ satisfies the inequality $d(h(x y)$, $h(x) h(y))<\delta$ for all $x, y \in G_{1}$, then there exists a homomorphism $H: G_{1} \rightarrow$ $G_{2}$ with $d(h(x), H(x))<\varepsilon$ for all $x \in G_{1}$ ?

The case of approximately additive mappings was solved by Hyers [3] under the assumption that $G_{1}$ and $G_{2}$ are Banach spaces. Later, the result of Hyers was significantly generalized by Rassias [11]. It should be remarked that we can find in [4] a lot of references concerning the stability of functional equations (see also $[2,5,6]$ ).

In $[12,13]$, Skof investigated the Hyers-Ulam stability of the additive functional equation for many cases of restricted domains in $\mathbb{R}$. Later, Losonczi [9] proved the local stability of the additive equation for more general cases and applied the result to the proof of stability of the Hosszú's functional equation.

In Section 2, the Hyers-Ulam stability of the additive equation will be investigated for a large class of unbounded domains. Moreover, in Section 3, we will apply the previous result to the proof of the local stability of the Jensen's functional equation on unbounded domains.

Throughout this paper, let $E_{1}$ and $E_{2}$ be a real (or complex) normed space and a Banach space, respectively.

2. Stability of additive equation on restricted domains. Assume that $\varphi$ : $(0, \infty) \rightarrow[0, \infty)$ is a decreasing mapping for which there exists a $d>0$ such 
that

$$
\varphi(s) \leq s,
$$

for any $s \geq d$.

We now define

$$
\begin{gathered}
B_{1}=\left\{(x, y) \in E_{1} \backslash\{0\} \times E_{1}:\|y\|<\varphi(\|x\|)\right\} \cup\left\{(0, y) \in E_{1}^{2}: y \in E_{1}\right\}, \\
B_{2}=\left\{(x, y) \in E_{1}^{2}:\|x+y\|<d\right\} .
\end{gathered}
$$

In the following theorem, we generalize the theorems of Skof $[12,13]$ and of Losonczi [9] concerning the stability of the additive equation on restricted domains.

THEOREM 2.1. If a mapping $f: E_{1} \rightarrow E_{2}$ with $\|f(0)\| \leq \varepsilon$ satisfies the inequality

$$
\|f(x+y)-f(x)-f(y)\| \leq \varepsilon
$$

for some $\varepsilon \geq 0$ and all $(x, y) \in E_{1}^{2} \backslash\left(B_{1} \cup B_{2}\right)$, then there exists a unique additive mapping $A: E_{1} \rightarrow E_{2}$ such that

$$
\|f(x)-A(x)\| \leq 39 \varepsilon, \quad \forall x \in E_{1} .
$$

Proof. First, we assume that $(x, y) \in B_{2}$ satisfies $x \neq 0, y \neq 0$, and $x+y \neq$ 0 . For this case, we can choose a $z_{1} \in E_{1}$ with

$$
\begin{gathered}
\left\|z_{1}\right\| \geq \varphi(\|x+y\|), \quad\left\|z_{1}\right\| \geq \varphi(\|x\|), \quad\left\|x+z_{1}\right\| \geq \varphi(\|y\|), \\
\left\|x+y+z_{1}\right\| \geq d, \quad\left\|x+z_{1}\right\| \geq d .
\end{gathered}
$$

Thus, the pairs $\left(x+y, z_{1}\right),\left(x, z_{1}\right)$, and $\left(y, x+z_{1}\right)$ do not belong to $B_{1} \cup B_{2}$. Hence, it follows from (2.3) that

$$
\begin{aligned}
\|f(x+y)-f(x)-f(y)\| \leq & \left\|-f\left(x+y+z_{1}\right)+f(x+y)+f\left(z_{1}\right)\right\| \\
& +\left\|f\left(x+z_{1}\right)-f(x)-f\left(z_{1}\right)\right\| \\
& +\left\|f\left(x+y+z_{1}\right)-f(y)-f\left(x+z_{1}\right)\right\| \\
\leq & 3 \varepsilon,
\end{aligned}
$$

for any $(x, y) \in B_{2}$ with $x \neq 0, y \neq 0$, and $x+y \neq 0$.

When $x=0$ or $y=0$, we have

$$
\|f(x+y)-f(x)-f(y)\|=\|f(0)\| \leq \varepsilon .
$$

Taking this fact into account, we see that inequality (2.6) is valid for all $(x, y) \in$ $B_{2}$ with $x+y \neq 0$. 
We now assume that $(x, y) \in B_{2}$ satisfies $x+y=0$ and $\|x\| \geq d$. (In this case, $\|y\|=\|-x\| \geq d$.) In view of (2.1), both the pairs $(-x,-x)$ and $(x,-2 x)$ do not belong to $B_{1} \cup B_{2}$. Hence, it follows from (2.3) that

$$
\|f(-2 x)-2 f(-x)\| \leq \varepsilon, \quad\|f(-x)-f(x)-f(-2 x)\| \leq \varepsilon .
$$

From the last two inequalities we get

$$
\begin{aligned}
\|f(x+y)-f(x)-f(y)\|= & \|f(0)-f(x)-f(-x)\| \\
\leq & \|f(0)\|+\|f(-2 x)-2 f(-x)\| \\
& +\|f(-x)-f(x)-f(-2 x)\| \\
\leq & 3 \varepsilon .
\end{aligned}
$$

Considering all the previous inequalities including (2.3), we conclude that $f$ satisfies the inequality

$$
\|f(x+y)-f(x)-f(y)\| \leq 3 \varepsilon
$$

for all $(x, y) \in E_{1}^{2} \backslash\left(B_{1} \cup B_{2}\right) \cup\left\{(u, v) \in B_{2}:\|u\| \geq d\right\}$.

Now, let $(x, y) \in E_{1}^{2}$ be arbitrarily given with $\|x\| \geq d$ and $\|y\| \geq d$. Since $\varphi$ is decreasing, we see by (2.1) that

$$
\varphi(\|x\|) \leq \varphi(d) \leq d \leq\|y\|
$$

and this implies that $(x, y) \notin B_{1}$. If, moreover, the given pair $(x, y)$ belongs to $B_{2}$, then $(x, y) \in\left\{(u, v) \in B_{2}:\|u\| \geq d\right\}$. Otherwise, $(x, y) \in E_{1}^{2} \backslash\left(B_{1} \cup B_{2}\right)$. Hence, it follows from (2.10) that

$$
\|f(x+y)-f(x)-f(y)\| \leq 3 \varepsilon
$$

for all $(x, y) \in E_{1}^{2}$ with $\|x\| \geq d$ and $\|y\| \geq d$.

Assume that $(x, y) \in E_{1}^{2}$ with $\|x\|<d$ and $\|y\| \geq 4 d$. In this case, we may choose a $z_{2} \in E_{1}$ with $2 d \leq\left\|z_{2}\right\|<3 d$. Then, it holds that

$$
\begin{aligned}
& \left\|x-z_{2}\right\| \geq d, \quad\left\|y+z_{2}\right\| \geq d, \quad\left\|x-z_{2}\right\| \geq d, \quad\left\|z_{2}\right\| \geq 2 d, \\
& \left\|-z_{2}\right\| \geq 2 d, \quad\left\|y+z_{2}\right\| \geq d, \quad\left\|z_{2}\right\| \geq 2 d, \quad\left\|-z_{2}\right\| \geq 2 d \text {. }
\end{aligned}
$$


It then follows from (2.12) and (2.13) that

$$
\begin{aligned}
\|f(x+y)-f(x)-f(y)\| \leq & \left\|f(x+y)-f\left(x-z_{2}\right)-f\left(y+z_{2}\right)\right\| \\
& +\left\|-f(x)+f\left(x-z_{2}\right)+f\left(z_{2}\right)\right\| \\
& +\left\|-f(y)+f\left(-z_{2}\right)+f\left(y+z_{2}\right)\right\| \\
& +\left\|f(0)-f\left(z_{2}\right)-f\left(-z_{2}\right)\right\|+\|-f(0)\| \\
\leq & 13 \varepsilon,
\end{aligned}
$$

for $(x, y) \in E_{1}^{2}$ with $\|x\|<d$ and $\|y\| \geq 4 d$.

Combining (2.12) and (2.14), we have

$$
\|f(x+y)-f(x)-f(y)\| \leq 13 \varepsilon
$$

for all $(x, y) \in E_{1}^{2}$ with $\|y\| \geq 4 d$. Since the Cauchy difference $f(x+y)-f(x)-$ $f(y)$ is symmetric with respect to $x$ and $y$, we conclude that inequality (2.15) is true for all $(x, y) \in E_{1}^{2}$ with $\|x\| \geq 4 d$ or $\|y\| \geq 4 d$.

If $(x, y) \in E_{1}^{2}$ satisfies $\|x\|<4 d$ and $\|y\|<4 d$, then we can choose a $z_{3} \in E_{1}$ with $\left\|z_{3}\right\| \geq 8 d$. Then, we have $\left\|x+z_{3}\right\| \geq 4 d$. Since inequality (2.15) holds true for all $(x, y) \in E_{1}^{2}$ with $\|x\| \geq 4 d$ or $\|y\| \geq 4 d$, we get

$$
\begin{aligned}
\|f(x+y)-f(x)-f(y)\| \leq & \left\|-f\left(x+y+z_{3}\right)+f(x+y)+f\left(z_{3}\right)\right\| \\
& +\left\|f\left(x+z_{3}\right)-f(x)-f\left(z_{3}\right)\right\| \\
& +\left\|f\left(x+y+z_{3}\right)-f(y)-f\left(x+z_{3}\right)\right\| \\
\leq & 39 \varepsilon,
\end{aligned}
$$

for any $(x, y) \in E_{1}^{2}$ with $\|x\|<4 d$ and $\|y\|<4 d$.

Inequality (2.16) together with (2.15) yields

$$
\|f(x+y)-f(x)-f(y)\| \leq 39 \varepsilon, \quad \forall x, y \in E_{1} .
$$

According to [1], there exists a unique additive mapping $A: E_{1} \rightarrow E_{2}$ that satisfies inequality (2.4) for each $x$ in $E_{1}$.

COROLlary 2.2. Let $d>0$ and $\varepsilon \geq 0$ be given. If a mapping $f: E_{1} \rightarrow E_{2}$ with $\|f(0)\| \leq \varepsilon$ satisfies inequality (2.3) for all $x, y \in E_{1}$ with $\max \{\|x\|,\|y\|\} \geq d$ and $\|x+y\| \geq d$, then there exists a unique additive mapping $A: E_{1} \rightarrow E_{2}$ that satisfies inequality (2.4) for each $x \in E_{1}$.

Proof. Because of the symmetry property of the Cauchy difference with respect to $x$ and $y$, we can, without loss of generality, assume that $f$ satisfies inequality (2.3) for all $x, y \in E_{1}$ with $\|y\| \geq d$ and $\|x+y\| \geq d$. 
For a constant mapping $\varphi(s)=d(s>0)$, define

$$
\begin{gathered}
B_{1}=\left\{(x, y) \in E_{1} \backslash\{0\} \times E_{1}:\|y\|<d\right\} \cup\left\{(0, y) \in E_{1}^{2}: y \in E_{1}\right\}, \\
B_{2}=\left\{(x, y) \in E_{1}^{2}:\|x+y\|<d\right\} .
\end{gathered}
$$

Since

$$
\begin{aligned}
& E_{1}^{2} \backslash B_{1}=\left\{(x, y) \in E_{1} \backslash\{0\} \times E_{1}:\|y\| \geq d\right\}, \\
& E_{1}^{2} \backslash B_{2}=\left\{(x, y) \in E_{1}^{2}:\|x+y\| \geq d\right\},
\end{aligned}
$$

we have

$$
E_{1}^{2} \backslash\left(B_{1} \cup B_{2}\right)=\left\{(x, y) \in E_{1} \backslash\{0\} \times E_{1}:\|y\| \geq d \text { and }\|x+y\| \geq d\right\} .
$$

Thus, it follows from our hypothesis that $f$ satisfies inequality (2.3) for all $(x, y) \in E_{1}^{2} \backslash\left(B_{1} \cup B_{2}\right)$.

According to Theorem 2.1, there exists a unique additive mapping $A: E_{1} \rightarrow$ $E_{2}$ that satisfies inequality (2.4) for all $x \in E_{1}$.

In 1983, Skof [12] presented an interesting asymptotic behavior of the additive mappings: a mapping $f: \mathbb{R} \rightarrow \mathbb{R}$ is additive if and only if $\mid f(x+y)-f(x)-$ $f(y) \mid \rightarrow 0$ as $|x|+|y| \rightarrow \infty$.

Without difficulty, the above theorem of Skof can be extended to mappings from a real normed space to a Banach space. We now apply Corollary 2.2 to a generalization of Skof theorem.

COROLLARY 2.3. A mapping $f: E_{1} \rightarrow E_{2}$ is additive if and only if

$$
\|f(x+y)-f(x)-f(y)\| \longrightarrow 0
$$

as $\|x+y\| \rightarrow \infty$.

Proof. On account of the hypothesis, there exists a decreasing sequence $\left(\varepsilon_{n}\right)$ with $\lim _{n \rightarrow \infty} \varepsilon_{n}=0$ and

$$
\|f(x+y)-f(x)-f(y)\| \leq \varepsilon_{n},
$$

for all $(x, y) \in E_{1}^{2}$ with $\|x+y\| \geq n$. With $y=0$ and $\|x\| \rightarrow \infty$, our hypothesis implies that $f(0)=0$.

By Corollary 2.2, there exists a unique additive mapping $A_{n}: E_{1} \rightarrow E_{2}$ such that

$$
\left\|f(x)-A_{n}(x)\right\| \leq 39 \varepsilon_{n}, \quad \forall x \in E_{1} .
$$

Now, let $l$ and $m$ be integers with $m>l>0$. Then, inequality (2.23) implies that

$$
\left\|f(x)-A_{m}(x)\right\| \leq 39 \varepsilon_{m} \leq 39 \varepsilon_{l},
$$


for $x \in E_{1}$, and further, the uniqueness of $A_{n}$ implies that $A_{m}=A_{l}$ for all integers $l, m>0$, that is, $A_{n}=A_{1}$ for any $n \in \mathbb{N}$. By letting $m \rightarrow \infty$ in the last inequality, we get

$$
\left\|f(x)-A_{1}(x)\right\|=0
$$

for any $x \in E_{1}$, which means that $f$ is additive. The reverse assertion is trivial.

3. Stability of Jensen's equation on restricted domains. Kominek investigated in [8] the Hyers-Ulam stability of the Jensen's functional equation

$$
2 f\left(\frac{x+y}{2}\right)=f(x)+f(y)
$$

for the class of mappings defined on a bounded subset of $\mathbb{R}^{N}$. On the other hand, the author proved in [7] the Hyers-Ulam stability of that equation on unbounded domains.

In this section, we use Theorem 2.1 to generalize the theorems of the author and of Kominek.

Let $\varphi_{1}:[0, \infty) \rightarrow[0, \infty)$ be a decreasing mapping that satisfies $\varphi_{1}(0)=d_{0}>$ 0. Define

$$
\begin{aligned}
& B_{1}=\left\{(x, y) \in E_{1} \backslash\{0\} \times E_{1}:\|y\|<\varphi_{1}(\|x\|)\right\} \cup\left\{(0, y) \in E_{1}^{2}: y \in E_{1}\right\}, \\
& B_{2}=\left\{(x, y) \in E_{1}^{2}:\|x+y\|<d_{0}\right\}, \\
& D=\left\{(0, y) \in E_{1}^{2}:\|y\| \geq d_{0}\right\} .
\end{aligned}
$$

THEOREM 3.1. If a mapping $f: E_{1} \rightarrow E_{2}$ satisfies the inequality

$$
\left\|2 f\left(\frac{x+y}{2}\right)-f(x)-f(y)\right\| \leq \varepsilon
$$

for some $\varepsilon \geq 0$ and all $(x, y) \in E_{1}^{2} \backslash\left(B_{1} \cup B_{2}\right) \cup D$, then there exists a unique additive mapping $A: E_{1} \rightarrow E_{2}$ such that

$$
\|f(x)-A(x)-f(0)\| \leq 78 \varepsilon
$$

for any $x \in E_{1}$.

Proof. If we substitute $g(x)$ for $f(x)-f(0)$ in (3.3), then

$$
\left\|2 g\left(\frac{x+y}{2}\right)-g(x)-g(y)\right\| \leq \varepsilon,
$$


for any $(x, y) \in E_{1}^{2} \backslash\left(B_{1} \cup B_{2}\right) \cup D$. With $x=0$ and $\|y\| \geq d_{0}$, inequality (3.5) yields

$$
\left\|2 g\left(\frac{y}{2}\right)-g(y)\right\| \leq \varepsilon
$$

for each $y \in E_{1}$ with $\|y\| \geq d_{0}$. Replace $y$ by $x+y\left(\|x+y\| \geq d_{0}\right)$ in inequality (3.6) to get

$$
\left\|2 g\left(\frac{x+y}{2}\right)-g(x+y)\right\| \leq \varepsilon
$$

for all $x, y \in E_{1}$ with $\|x+y\| \geq d_{0}$.

It follows from (3.5) and (3.7) that

$$
\begin{aligned}
\| g(x+y)- & g(x)-g(y) \| \\
& \leq\left\|g(x+y)-2 g\left(\frac{x+y}{2}\right)\right\|+\left\|2 g\left(\frac{x+y}{2}\right)-g(x)-g(y)\right\| \\
& \leq 2 \varepsilon,
\end{aligned}
$$

for every $(x, y) \in E_{1}^{2} \backslash\left(B_{1} \cup B_{2}\right) \cup D$ with $\|x+y\| \geq d_{0}$. Since $(x, y) \in E_{1}^{2} \backslash\left(B_{1} \cup\right.$ $B_{2}$ ) implies that $\|x+y\| \geq d_{0}$, the mapping $g$ surely satisfies

$$
\|g(x+y)-g(x)-g(y)\| \leq 2 \varepsilon,
$$

for all $(x, y) \in E_{1}^{2} \backslash\left(B_{1} \cup B_{2}\right)$.

It trivially holds that $\varphi_{1}(s) \leq s$ for all $s \geq d_{0}$. On account of Theorem 2.1, there exists a unique additive mapping $A: E_{1} \rightarrow E_{2}$ such that

$$
\|g(x)-A(x)\| \leq 78 \varepsilon
$$

for each $x$ in $E_{1}$.

Let $\varphi_{2}:(0, \infty) \rightarrow[0, \infty)$ be a continuous and decreasing mapping that satisfies

$$
0<d=\inf \left\{s>0: \varphi_{2}(s)=0\right\}<\infty .
$$

Furthermore, assume that the restriction $\left.\varphi_{2}\right|_{(0, d]}$ is strictly decreasing. 
Now, we define

$$
\begin{aligned}
B_{1} & =\left\{(x, y) \in E_{1} \backslash\{0\} \times E_{1}:\|y\|<\varphi_{2}(\|x\|)\right\} \cup\left\{(0, y) \in E_{1}^{2}: y \in E_{1}\right\}, \\
B_{2} & =\left\{(x, y) \in E_{1}^{2}:\|x+y\|<d_{0}\right\}, \\
D & =\left\{(0, y) \in E_{1}^{2}:\|y\| \geq d_{0}\right\},
\end{aligned}
$$

where we set $d_{0}=\inf \left\{d, \lim _{s \rightarrow 0+} \varphi_{2}(s)\right\}$.

COROLLARY 3.2. If a mapping $f: E_{1} \rightarrow E_{2}$ satisfies inequality (3.3) for some $\varepsilon \geq 0$ and all $(x, y) \in E_{1}^{2} \backslash\left(B_{1} \cup B_{2}\right) \cup D$, then there exists a unique additive mapping $A: E_{1} \rightarrow E_{2}$ satisfying inequality (3.4) for all $x \in E_{1}$.

Proof. First, we define a mapping $\varphi_{0}:[0, \infty) \rightarrow[0, \infty)$ by

$$
\varphi_{0}(s)= \begin{cases}d_{0}, & \text { for } s=0, \\ \inf \left\{\varphi_{2}(s), \inf \varphi_{2}^{-1}(s)\right\}, & \text { for } s>0,\end{cases}
$$

where we set $\varphi_{2}^{-1}(t)=\left\{s>0: \varphi_{2}(s)=t\right\}$ and $\inf \varnothing=\infty$. (We cannot exclude the case $\varphi_{2}^{-1}(s)=\varnothing$ from the above definition.) We define

$$
\begin{aligned}
\tilde{B}_{1} & =\left\{(x, y) \in E_{1} \backslash\{0\} \times E_{1}:\|y\|<\varphi_{0}(\|x\|)\right\} \cup\left\{(0, y) \in E_{1}^{2}: y \in E_{1}\right\}, \\
\tilde{B}_{2} & =\left\{(x, y) \in E_{1}^{2}:\|x+y\|<d_{0}\right\}, \\
\tilde{D} & =\left\{(0, y) \in E_{1}^{2}:\|y\| \geq d_{0}\right\} .
\end{aligned}
$$

The fact that $\varphi_{0}(s) \leq \varphi_{2}(s)$ for all $s>0$ implies that $\tilde{B}_{1} \subset B_{1}$. Since $B_{2}=\tilde{B}_{2}$ and $D=\tilde{D}$, we get

$$
E_{1}^{2} \backslash\left(B_{1} \cup B_{2}\right) \cup D \subset E_{1}^{2} \backslash\left(\tilde{B}_{1} \cup \tilde{B}_{2}\right) \cup \tilde{D}
$$

Now, assume that $(x, y) \in E_{1}^{2} \backslash\left(\tilde{B}_{1} \cup \tilde{B}_{2}\right) \cup \tilde{D}$ but $(x, y) \notin E_{1}^{2} \backslash\left(B_{1} \cup B_{2}\right) \cup D$. Because $(x, y) \notin D$ and $(x, y) \notin B_{2}$, we have

$$
x \neq 0, \quad\|x+y\| \geq d_{0} .
$$

Moreover, $(x, y)$ should belong to $B_{1} \backslash \tilde{B}_{1}$, that is,

$$
0<\inf \varphi_{2}^{-1}(\|x\|) \leq\|y\|<\varphi_{2}(\|x\|)
$$


(Since $\|x\|>0$ and $\left.\varphi_{2}\right|_{(0, d]}$ is strictly decreasing, we have inf $\varphi_{2}^{-1}(\|x\|)>0$.) If we assume that $(y, x) \in B_{1}$, then we get $\|x\|<\varphi_{2}(\|y\|)$. This fact implies that $\|y\|<\inf \varphi_{2}^{-1}(\|x\|)$, which is contrary to (3.17). Hence, by (3.16), we conclude that $(y, x) \notin B_{1} \cup B_{2}$. This fact together with (3.3), yields

$$
\left\|2 f\left(\frac{y+x}{2}\right)-f(y)-f(x)\right\| \leq \varepsilon
$$

for all $(x, y) \in E_{1}^{2} \backslash\left(\tilde{B}_{1} \cup \tilde{B}_{2}\right) \cup \tilde{D}$.

We now define another mapping $\varphi:[0, \infty) \rightarrow[0, \infty)$ by

$$
\varphi(s)= \begin{cases}d_{0}, & \text { for } s=0, \\ \inf \left\{\varphi_{2}(s), \inf \varphi_{2}^{-1}(s)\right\}, & \text { for } 0<s \leq d_{1}, \\ \sup \left\{\varphi_{2}(s), \sup \varphi_{2}^{-1}(s)\right\}, & \text { for } s>d_{1},\end{cases}
$$

where $d_{1}>0$ is the unique fixed point of $\varphi_{2}$, that is, $d_{1}=\varphi_{2}\left(d_{1}\right)$, and we set $\inf \varnothing=\infty$ and $\sup \varnothing=0$.

Let $s_{i}>0(i=1,2,3,4)$ be arbitrarily given with $0<s_{1}<s_{2} \leq d_{1}<s_{3}<s_{4}$. Since $\varphi_{2}$ is decreasing, we have

$$
\begin{gathered}
\lim _{s \rightarrow 0+} \varphi_{2}(s) \geq \varphi_{2}\left(s_{1}\right) \geq \varphi_{2}\left(s_{2}\right) \geq d_{1} \geq \varphi_{2}\left(s_{3}\right) \geq \varphi_{2}\left(s_{4}\right), \\
d \geq \inf \varphi_{2}^{-1}\left(s_{1}\right) \geq \inf \varphi_{2}^{-1}\left(s_{2}\right) \geq d_{1} \geq \sup \varphi_{2}^{-1}\left(s_{3}\right) \geq \sup \varphi_{2}^{-1}\left(s_{4}\right) .
\end{gathered}
$$

Hence, we get

$$
\varphi(0) \geq \varphi\left(s_{1}\right) \geq \varphi\left(s_{2}\right) \geq \varphi\left(s_{3}\right) \geq \varphi\left(s_{4}\right)
$$

which implies that $\varphi$ is decreasing.

Similarly as before, we define

$$
\begin{aligned}
\hat{B}_{1} & =\left\{(x, y) \in E_{1} \backslash\{0\} \times E_{1}:\|y\|<\varphi(\|x\|)\right\} \cup\left\{(0, y) \in E_{1}^{2}: y \in E_{1}\right\}, \\
\hat{B}_{2} & =\left\{(x, y) \in E_{1}^{2}:\|x+y\|<d_{0}\right\}, \\
\hat{D} & =\left\{(0, y) \in E_{1}^{2}:\|y\| \geq d_{0}\right\} .
\end{aligned}
$$

Since $\hat{B}_{1} \supset \tilde{B}_{1}, \hat{B}_{2}=\tilde{B}_{2}$, and $\hat{D}=\tilde{D}$, we may conclude that inequality (3.3) holds true for all $(x, y) \in E_{1}^{2} \backslash\left(\hat{B}_{1} \cup \hat{B}_{2}\right) \cup \hat{D}$.

According to Theorem 3.1, there exists a unique additive mapping $A: E_{1} \rightarrow$ $E_{2}$ such that inequality (3.4) is true for any $x \in E_{1}$.

The author in [7] proved that it needs only to show an asymptotic property of the Jensen difference to identify a given mapping with an additive one. 
Let $X$ and $Y$ be a real normed space and a real Banach space, respectively. A mapping $f: X \rightarrow Y$ with $f(0)=0$ is additive if and only if

$$
\left\|2 f\left(\frac{x+y}{2}\right)-f(x)-f(y)\right\| \rightarrow 0
$$

as $\|x\|+\|y\| \rightarrow \infty$.

By using Theorem 3.1, we now prove an asymptotic behavior of additive mappings which generalizes the above result.

COROLLARY 3.3. A mapping $f: E_{1} \rightarrow E_{2}$ with $f(0)=0$ is additive if and only if

$$
\left\|2 f\left(\frac{x+y}{2}\right)-f(x)-f(y)\right\| \rightarrow 0
$$

as $\|x+y\| \rightarrow \infty$.

Proof. According to our hypothesis, there exists a decreasing sequence $\left(\varepsilon_{n}\right)$ with $\lim _{n \rightarrow \infty} \varepsilon_{n}=0$ and

$$
\left\|2 f\left(\frac{x+y}{2}\right)-f(x)-f(y)\right\| \leq \varepsilon_{n},
$$

for all $(x, y) \in E_{1}^{2}$ with $\|x+y\| \geq n$.

The mapping $\varphi_{1}:[0, \infty) \rightarrow[0, \infty)$ defined by $\varphi_{1}(s)=-s+n(s \geq 0)$ is decreasing. Moreover, it holds that $\varphi_{1}(0)=n$. We define

$$
\begin{aligned}
& B_{1}=\left\{(x, y) \in E_{1} \backslash\{0\} \times E_{1}:\|y\|<-\|x\|+n\right\} \cup\left\{(0, y) \in E_{1}^{2}: y \in E_{1}\right\}, \\
& B_{2}=\left\{(x, y) \in E_{1}^{2}:\|x+y\|<n\right\}, \\
& D=\left\{(0, y) \in E_{1}^{2}:\|y\| \geq n\right\} .
\end{aligned}
$$

Since $B_{1} \cup B_{2}=\left\{(x, y) \in E_{1}^{2}: x=0\right.$ or $\left.\|x+y\|<n\right\}$ and $D=\left\{(x, y) \in E_{1}^{2}\right.$ : $x=0$ and $\|x+y\| \geq n\}$, we have

$$
E_{1}^{2} \backslash\left(B_{1} \cup B_{2}\right)=\left\{(x, y) \in E_{1}^{2}: x \neq 0 \text { and }\|x+y\| \geq n\right\}
$$

and hence

$$
E_{1}^{2} \backslash\left(B_{1} \cup B_{2}\right) \cup D=\left\{(x, y) \in E_{1}^{2}:\|x+y\| \geq n\right\} .
$$

Therefore, inequality (3.25) holds true for all $(x, y) \in E_{1}^{2} \backslash\left(B_{1} \cup B_{2}\right) \cup D$. 
According to Theorem 3.1, there exists a unique additive mapping $A_{n}: E_{1} \rightarrow$ $E_{2}$ such that

$$
\left\|f(x)-A_{n}(x)\right\| \leq 78 \varepsilon_{n}, \quad \forall x \in E_{1} .
$$

Now, let $l$ and $m$ be positive integers with $m>l$. Then, it follows from (3.29) that

$$
\left\|f(x)-A_{m}(x)\right\| \leq 78 \varepsilon_{m} \leq 78 \varepsilon_{l},
$$

for $x \in E_{1}$. However, the uniqueness of $A_{n}$ implies that $A_{m}=A_{l}$ for all positive integers $l$ and $m$, that is, $A_{n}=A_{1}$ for any $n \in \mathbb{N}$. By letting $m \rightarrow \infty$ in the last inequality, we get

$$
\left\|f(x)-A_{1}(x)\right\|=0
$$

for each $x \in E_{1}$, which implies that $f$ is an additive mapping.

The reverse assertion is trivial because every additive mapping $f: E_{1} \rightarrow E_{2}$ is a solution of the Jensen functional equation (see [10]).

ACKNOWLEDgment. The first author was supported by Korea Research Foundation grant KRF-DP0031.

\section{REFERENCES}

[1] G. L. Forti, The stability of homomorphisms and amenability, with applications to functional equations, Abh. Math. Sem. Univ. Hamburg 57 (1987), 215-226.

[2] _ _ Hyers-Ulam stability of functional equations in several variables, Aequationes Math. 50 (1995), no. 1-2, 143-190.

[3] D. H. Hyers, On the stability of the linear functional equation, Proc. Nat. Acad. Sci. U.S.A. 27 (1941), 222-224.

[4] D. H. Hyers, G. Isac, and T. M. Rassias, Stability of Functional Equations in Several Variables, Progress in Nonlinear Differential Equations and Their Applications, vol. 34, Birkhäuser Boston, Massachusetts, 1998.

[5] D. H. Hyers and T. M. Rassias, Approximate homomorphisms, Aequationes Math. 44 (1992), no. 2-3, 125-153.

[6] S.-M. Jung, Hyers-Ulam-Rassias stability of functional equations, Dynam. Systems Appl. 6 (1997), no. 4, 541-565.

[7] __ Hyers-Ulam-Rassias stability of Jensen's equation and its application, Proc. Amer. Math. Soc. 126 (1998), no. 11, 3137-3143.

[8] Z. Kominek, On a local stability of the Jensen functional equation, Demonstratio Math. 22 (1989), no. 2, 499-507.

[9] L. Losonczi, On the stability of Hosszú's functional equation, Results Math. 29 (1996), no. 3-4, 305-310.

[10] J. C. Parnami and H. L. Vasudeva, On Jensen's functional equation, Aequationes Math. 43 (1992), no. 2-3, 211-218.

[11] T. M. Rassias, On the stability of the linear mapping in Banach spaces, Proc. Amer. Math. Soc. 72 (1978), no. 2, 297-300.

[12] F. Skof, Local properties and approximation of operators, Rend. Sem. Mat. Fis. Milano 53 (1983), 113-129 (Italian). 
[13] - On the approximation of locally $\delta$-additive mappings, Atti Accad. Sci. Torino Cl. Sci. Fis. Mat. Natur. 117 (1983), no. 4-6, 377-389 (Italian).

[14] S. M. Ulam, Problems in Modern Mathematics, John Wiley \& Sons, New York, 1964.

Soon-Mo Jung: Mathematics Section, College of Science and Technology, Hong-Ik University, 339-701 Chochiwon, Korea

E-mail address: smjung@wow.hongik.ac.kr

Byungbae Kim: Mathematics Section, College of Science and Technology, Hong-Ik University, 339-701 Chochiwon, Korea

E-mail address: bkim@wow.hongik.ac.kr 


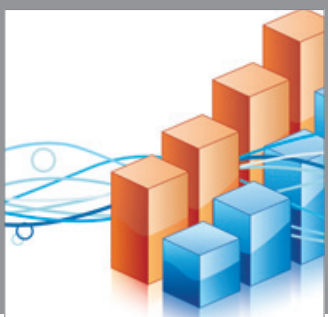

Advances in

Operations Research

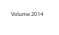

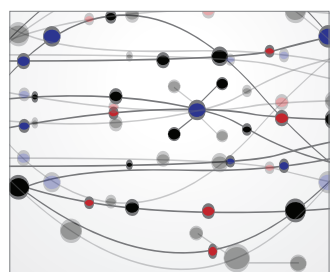

\section{The Scientific} World Journal
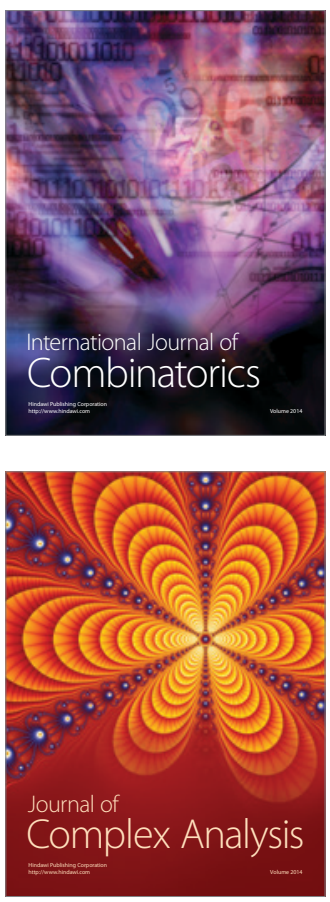

International Journal of

Mathematics and

Mathematical

Sciences
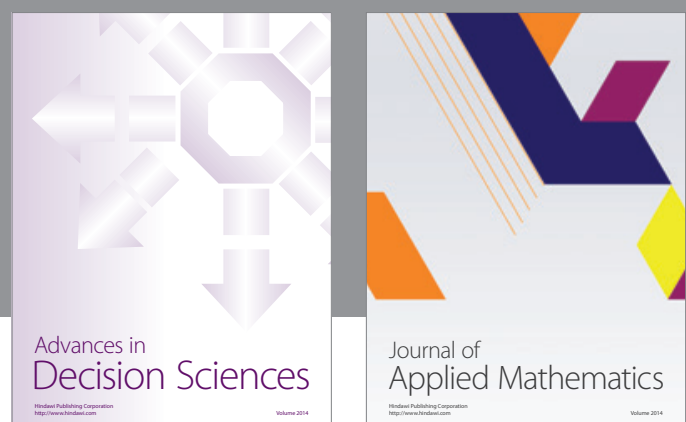

Journal of

Applied Mathematics
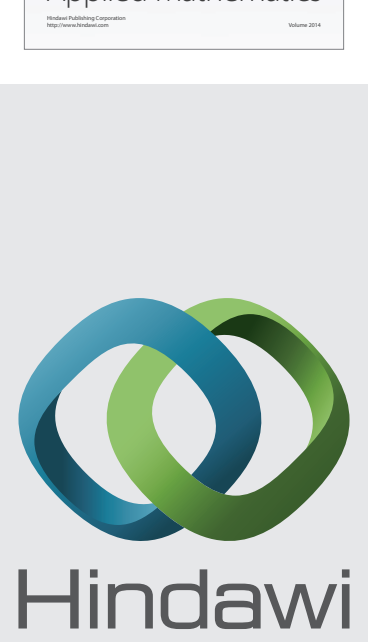

Submit your manuscripts at http://www.hindawi.com
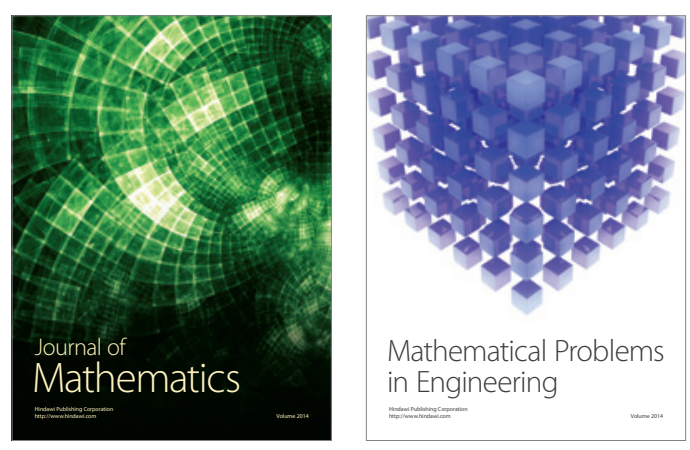

Mathematical Problems in Engineering
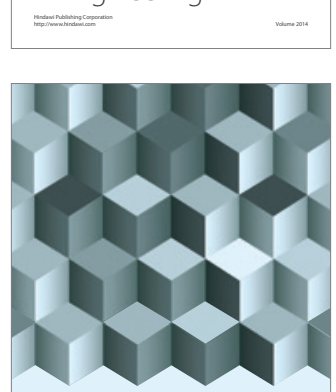

Journal of

Function Spaces
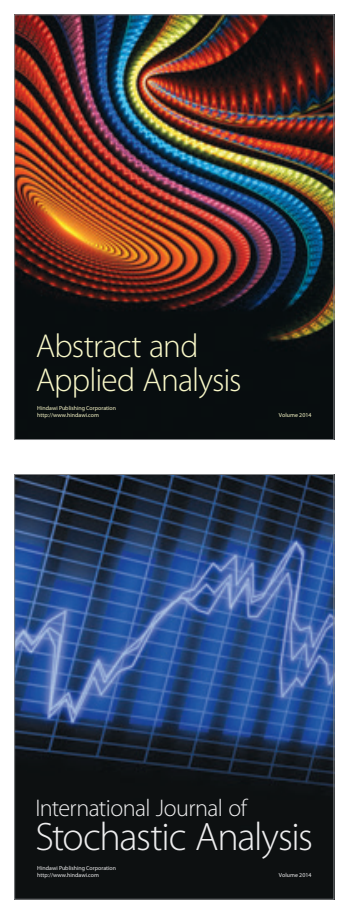

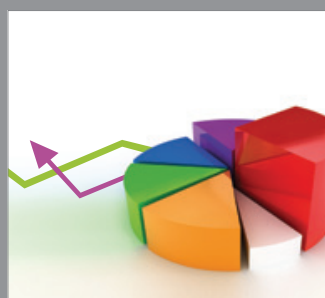

ournal of

Probability and Statistics

Promensencen
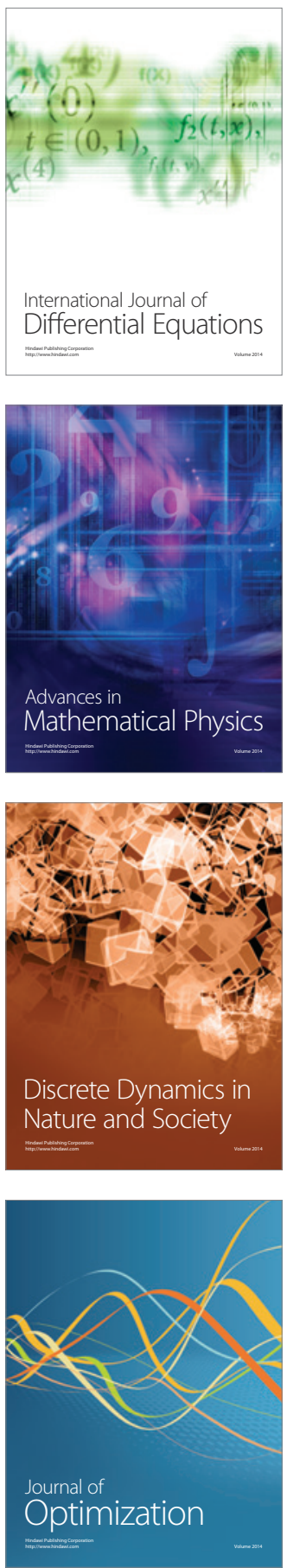